\title{
Human papillomavirus-related psychosocial impact of patients with genital warts in China: a hospital-based cross-sectional study
}

Shu-Zhen Qi ${ }^{1+}$, Shao-Ming Wang ${ }^{2 \dagger}$, Ju-Fang Shi ${ }^{2}$, Qian-Qiu Wang ${ }^{1,3}$, Xiang-Sheng Chen ${ }^{1,3}$, Li-Jun Sun ${ }^{4}$, An Liu ${ }^{4}$, Nanci Zhang ${ }^{5}$, Ning Jiang ${ }^{1,3}$, Priya Siva ${ }^{2,6}$, Xiu-Lian $X^{1}$ and You-Lin Qiao ${ }^{2^{*}}$

\begin{abstract}
Background: Genital warts (GW) are the most common sexually transmitted infections. To date, few studies using a human papillomavirus (HPV)-specific questionnaire have focused on the impact of quality of life (QoL) among patients with GW in developing countries. The origins of GW related psychosocial burdens and variations between genders were poorly characterized as well.

Methods: A hospital-based survey was conducted in Beijing and Nanjing of China in 2008. Eligible patients aged 18-65 who had a diagnosis of GW within 3 months were recruited. Demographic information, HPV knowledge, and assessment of psychosocial burden were collected by the HPV Impact Profile (HIP). The HIP examined 7 specific psychosocial domains by 29 items: (1) worries and concerns, (2) emotional impact, (3) sexual impact, (4) self-image, (5) partner and transmission, (6) interactions with physicians, and (7) control/life impact. HIP scores are reversely relates to the subjects' QoL, by which a higher score indicating a heavier psychosocial burden.

Results: Patients with GW experienced heavier psychosocial burdens than those of the general population, and females experienced heavier burdens than males (male vs. female: 49.20 vs.51.38, $P<0.001$ ). "Self Image" and "Sexual Impact" were the two domains that affected patients the most, with mean HIP scores of 63.09 and 61.64, respectively. Women suffered heavier psychosocial burdens than men in the domain of "Worries and Concerns" (female vs. male: 54.57 vs. 42.62, $P<0.001$ ), but lower psychosocial burdens in the domains of "Sexual Impact" (female vs. male: 59.16 vs. 65.26, $P<0.001$ ) and "Interactions with Doctors" (female vs. male: 34.40 vs. 41.97, $P<0.001$ ). Patients from Nanjing suffered a higher psychosocial burden than those of Beijing, especially in domains of "Emotional Impact", "Sexual Impact", "Partner and Transmission", and "Interactions with Doctors".

Conclusions: Patients with GW suffered heavy psychological burden, and self-image and sexual-related concern were the primary cause of burdens. It's important to change the current biomedical model to bio-psycho-social model, and establish psychosocial support systems. The distinctions of origins of psychosocial burden between genders identified will be informative for prevention of GW and control efforts in China and other similar settings.
\end{abstract}

Keywords: Genital warts (GW), Psychosocial burden, Quality of Life (QoL), Human papillomavirus Impact Profile (HIP)

\footnotetext{
* Correspondence: qiaoy@cicams.ac.cn

${ }^{\dagger}$ Equal contributors

${ }^{2}$ Cancer Institute/Hospital, Chinese Academy of Medical Sciences, Peking

Union Medical College, Beijing, China

Full list of author information is available at the end of the article
} 


\section{Background}

Genital warts (GW) are the most common sexually transmitted Infections (STI) in the world with a substantial burden of disease. The reported incidence of GW in United States is 205/100,000 person-year [1]. This high incidence elicits concern regarding GW incidence in China, a developing country lacking complete disease surveillance and registration systems. GW are a highly visible and recurrent condition primarily caused by low risk Human Papillomavirus (HPV) infection, [2]. Timely prophylactic HPV vaccination has been shown to be an effective way to prevent precancerous lesions and GW [3]. With worldwide implementation of vaccination, the potential added benefits of preventing both the majority of cases of GW and accompanying psychosocial impact by HPV vaccination should be considered and assessed.

In addition to biological intervention, psychosocial intervention turns out to be an important area for the current disease prevention and control, and change from a biomedical model to a bio-psycho-social model. Previous studies demonstrated that GW was associated with a significant detriment to health-related Quality of life (QoL), but the origins of these psychosocial burdens were still unclear [4-7]. Recently, literature reported that female patients with GW underwent a significantly heavier psychosocial burden than the general population in China $[5,8]$. This national study highlighted the psychosocial burden of Chinese women with GW, inciting interest in exploring the afflictions of a different group with extremely high incidence, Chinese male GW patients, as well as variations between sexes.

Therefore, in order to address the HPV-related psychosocial burden of patients with GW for both genders, we conducted a hospital-based study in the provincial hospital of Beijing and Nanjing of China. We intend to thoroughly investigate differences in the origins of HPVrelated psychological burden between genders by using HPV impact profile (HIP), which is a standardized HPVspecific psychosocial questionnaire. Measures for alleviating emotional encumbrances are to be explored in this study as well. Thereafter, we aim to provide suggestions for future psychosocial intervention of sexual transmitted disease such as genital warts in China. For the purposes of this study, the term "psychosocial burden" will refer to the HPV-related impacts on QoL and psychology, which were assessed by HIP.

\section{Methods}

\section{Study design}

This study was part of a multi-center, hospital-based study in China focusing on HPV type distribution, psychosocial impacts, and cost-effectiveness of screening interventions $[5,8,9]$. As most patients with STIs are diagnosed in sexually transmitted diseases (STD) clinics in
China, subjects for this cross-sectional survey were recruited from the STD Clinic department of Institute of Dermatology in Nanjing and Beijing You An hospital in Beijing of China.

\section{Study population}

From February to May 2008, patients were invited to join the study during their routine clinical visits if they recently had a diagnosis of GW. It usually took half an hour to finish the visit by a deputy chief physician or 15-20 minutes by an attending doctor. After informed consent and a primary screening interview conducted by the investigator, patients were invited to attend the study regardless of their gender or whether they were an incident or recurrent case. Eligible subjects were aged 18-65 years, had experienced a hospital-based diagnosis of genital warts within the past three months, signed the informed consent, and were required to be in otherwise good physical health based on self-reported medical history with no significant co-morbid conditions. For the purpose of investigating the pure psychosocial impact of genital warts and to avoid confounding factors, patients were ineligible if they reported a history of HPV vaccination, alcohol or drug abuse in the past year, or other STIs over the past six months. Subjects were also ineligible if they were concurrently enrolled in other clinical studies, or had any condition that the investigator thought to be influential to the study.

Ethical approval was obtained from the Institutional Review Board of the Cancer Foundation of China.

\section{Data collection}

Basic demographic information (i.e., age, income, education, and marital status), HPV knowledge, and assessment of psychosocial burden were collected by the HIP questionnaire, $[5,10]$ which was completed by the patient in the presence of a trained interviewer who could assist with any questions participants had about the survey.

The HIP examined seven specific psychosocial domains by 29 items: 1) Worries and Concerns; 2) Emotional Impact; 3) Sexual Impact; 4) Self Image; 5) Partner and Transmission; 6) Interactions with Doctors; 7) Control/ Life Impact (see Table 1 and Additional file 1). The questionnaire administered to both sexes was identical, except for two questions directed at male participants regarding the gender of their sexual partners. Male subjects specified if their sexual activity was homosexual, heterosexual or both, and whether intercourse was vaginal, oral, anal, or a combination. Additionally, male subjects were not required to answer questions on the topic of suffering from cervical cancer.

The HIP questionnaire was demonstrated to have favorable reliability, construct validity, and ability to discriminate amongst varying degrees of disease severity 
Table 1 The domains and items of HPV Impact Profile (HIP) questionnaires

\begin{tabular}{lcc}
\hline Domain & Item examples & Items \\
\hline Worries and concerns & $\begin{array}{r}\text { Bad health condition, suffer from disease, having no treatment for disease, } \\
\text { loss of fertility, pain in the gynecologist visit }\end{array}$ & $7,12,13,15,16,17,18,19,20$ \\
Emotional impact & Unexpected result, depression, anxiety, angry, pessimistic & $2,3,5,8,14$ \\
Sexual impact & Frequency and satisfaction & 24,25 \\
Self image & Attractiveness, shame, disgust & $1,10,11,23$ \\
Partner and transmission & Partner acceptance, concern of transmission to/from partner & $9,21,22$ \\
Interactions with doctors & Pain, embarrassment or discomfort during clinical visit & $27,28,29$ \\
Control/life impact & Control of Health, concentration, sleep & $4,6,26$ \\
\hline
\end{tabular}

[10]. Cronbach's alpha ranged from 0.64 to 0.90 and was $\geq 0.7$ for $5 / 7$ domains [10]. The Mandarin Chinese version of the HIP questionnaire was generated through a standardized process, and has previously been used in Taiwan $[7,10,11]$.

Each item was scored individually to a 10-point discrete analog scale, which used visual-spatial, numeric, and verbal descriptive anchors (from "not at all" to "extremely") to assess the different psychosocial impact of each item on the participant. Each item's score was then linearly transformed to a $0-100$ scale according to the arithmetic of HIP [10]. Transformations were performed to unify the indicated meanings of individual scores (a higher score indicated heavier burden), with some items reverse-scored to account for the inverse meaning. The individual domain scale scores were computed as the sum of the items over the number of items answered in each associated domain. To create the overall HIP score, the mean was computed as the sum of all the items over the number of items answered on all domain scales. A mean total HIP score below 40 signified little to no impact; scores between 40 and 70 implied moderate impact; and above 70, indicated heavy psychosocial impact [7].

Quality control was performed throughout the project for uniformity. This included training local interviewers before the project started, providing all participants with a letter of introduction describing self-administration in order to obtain a more consistent standard for questionnaires, and running regular quality control procedures to ensure the completion of the questionnaires and accuracy of data entry.

\section{Data analysis}

The software SAS 9.0 was used for data analysis. Demographic information was compared using the Pearson $x^{2}$ test, except for age and HIP mean score which were estimated using $\mathrm{T}$ test analysis. GLM proc was used for the comparisons of HIP scores by different psychosocial domains between genders and areas. Histograms were used to visualize comparisons of psychosocial burdens between genders. All the analyses used two-sided tests.

\section{Results}

\section{Socio-demographic characteristics}

A total of 204 men and 334 women were invited to participate in this study. 13 men and 4 women refused to participate due to concern of privacy or time consumption. Finally, 330 eligible women and 191 men were enrolled. The majority of participants were married (66.2\%) with a mean age of 31.9 years old (yrs). In general, women were younger than men (Female vs. Male: 30.9 vs. 34.9 yrs, $P<0.001)$, had a lower level of education $(P<0.001)$ and income $(P<0.05)$. The coverage of insurance of women was lower than that of men as well (Female vs. Male: $34.5 \%$ vs. 52.0\%, $P<0.001$ ) (see Table 2). A quarter (25.8\%) of the participants were unaware of HPV and its effects.

In addition, for questions regarding nature of sexual partners, $98.5 \%$ of males were self-reported to be heterosexual. The percentages of male intercourse types were tabulated to be $88.7 \%, 8.8 \%$, and $2.5 \%$ for vaginal, vaginal associated with oral, and other combined variations (i.e. oral-anal, vaginal-anal), respectively.

\section{Psychosocial burden assessed by HIP}

Patients with genital warts suffered relatively high psychosocial burden, with a mean HIP score of 50.49 (95\% CI: 49.41-51.57). Generally, females experienced heavier psychosocial burden than males (male vs. female: 49.20 vs.51.38, $P<0.001)$.

Considering the sources of psychosocial burdens, "Self Image" and "Sexual Impact" were the two HPV-related domains that affected patients the most, with mean HIP scores of 63.09 and 61.64 , respectively, followed by "Worries and Concerns" (49.72), "Partner and Transmission" (48.42), "Control/Life Impact" (48.19), "Emotional Impact" (47.40), and "Interactions with Doctors" (37.47).

Comparing HIP domain scores between genders, significant differences were found in three domains. Women had heavier psychosocial burdens than men in domain of "Worries and Concerns" (female vs. male: 54.57 vs. 42.62, $P<0.001$ ), but lower psychosocial burdens in the domains of "Sexual Impact" (female vs. male: 59.16 vs. 65.26, $P<0.001$ ) and "Interactions with Doctors" (female vs. 
Table 2 Socio-demographic and major study variables for participants by gender

\begin{tabular}{|c|c|c|c|c|}
\hline Characteristics* & Total & Male & Female & $P$ Value \\
\hline $\mathrm{N}$ & 538 & 204 & 334 & \\
\hline Age $($ Mean $\pm S D)$ & $31.9 \pm 10.5$ & $34.9 \pm 10.7$ & $30.9 \pm 9.0$ & $<0.001$ \\
\hline \multicolumn{5}{|l|}{ Education } \\
\hline Junior High School and below & $113(21.0 \%)$ & $30(14.7 \%)$ & $83(24.8 \%)$ & \multirow[t]{3}{*}{0.008} \\
\hline Senior High School & $158(29.4 \%)$ & $58(28.4 \%)$ & 100 (29.9\%) & \\
\hline College and Above & 267 (49.6\%) & $116(56.9 \%)$ & $151(45.2 \%)$ & \\
\hline \multicolumn{5}{|l|}{ Monthly income (RMB, Yuan) } \\
\hline$<=800$ & $94(17.5 \%)$ & $25(12.3 \%)$ & $69(20.7 \%)$ & \multirow[t]{5}{*}{0.031} \\
\hline $801-1500$ & $68(12.6 \%)$ & $32(15.7 \%)$ & $36(10.8 \%)$ & \\
\hline $1501-3000$ & $191(35.5 \%)$ & $70(34.3 \%)$ & $121(36.2 \%)$ & \\
\hline$>3000$ & $184(34.2 \%)$ & $77(37.7 \%)$ & $107(32.0 \%)$ & \\
\hline Missing & $1(0.2 \%)$ & - & $1(0.3 \%)$ & \\
\hline Married (Yes) & $356(66.2 \%)$ & $144(70.6 \%)$ & $212(63.5 \%)$ & $>0.05$ \\
\hline insurance coverage & $221(41.2 \%)$ & $106(52.0 \%)$ & $115(34.5 \%)$ & $<0.001$ \\
\hline \multicolumn{5}{|l|}{ HPV knowledge } \\
\hline some & $109(20.3 \%)$ & $44(21.6 \%)$ & $65(19.5 \%)$ & \multirow[t]{4}{*}{$>0.05$} \\
\hline little & $290(53.9 \%)$ & $101(49.5 \%)$ & $189(56.6 \%)$ & \\
\hline none & $139(25.8 \%)$ & $59(28.9 \%)$ & $80(24.0 \%)$ & \\
\hline Heterosexual & - & $201(98.5 \%)$ & - & \\
\hline \multicolumn{5}{|l|}{ Sexual style } \\
\hline vaginal & $181(88.7 \%)$ & $181(88.7 \%)$ & - & \\
\hline vaginal + oral sex & $18(8.8 \%)$ & $18(8.8 \%)$ & - & \\
\hline others & $5(2.5 \%)$ & $5(2.5 \%)$ & - & \\
\hline HIP mean score $(95 \% \mathrm{Cl})$ & $50.49(49.41-51.57)$ & $49.20(47.46-50.94)$ & $51.38(49.95-52.80)$ & 0.007 \\
\hline
\end{tabular}

${ }^{*}$ Reported as $\mathrm{n}(\%), P$ value (2-sides) estimated using Chi-square test, except for age and HIP mean score, which were estimated using T-test. $\mathrm{Cl}$, Confidence interval.

male: 34.40 vs. 41.97, $P<0.001$ ) (see Figure 1). For domains of "Emotional Impact" (female vs. male: 47.58 vs. 47.14), "Self Image" (female vs. male: 63.31 vs. 62.77), "Control/Life Impact" (female vs. male: 48.35 vs. 47.96), and "Partner and Transmission" (female vs. male: 47.53 vs. 49.71) domains, females and males had comparable scores $(P>0.05)$ (see Table 3$)$.

We also compared the psychosocial burdens of patients from different settings. In general, patients from Nanjing showed a higher psychosocial burden than those of Beijing, especially in domains of "Emotional Impact" (Nanjing vs. Beijing: 52.85 vs. 44.59, $P<0.001$ ), "Sexual Impact" (Nanjing vs. Beijing: 70.49 vs. 57.07, $P<0.001)$, "Partner and Transmission" (Nanjing vs. Beijing: 56.38 vs. 44.31, $P<0.001$ ), and "Interactions with Doctors" (Nanjing vs. Beijing: 47.86 vs. 32.10, $P<0.001$ ) (see Figure 2).

\section{Discussion}

The current work is a unique study focusing on the HPV-related psychosocial burden of male and female patients with GW using the HPV-specific HIP questionnaire. Our results showed that patients with GW had a substantially higher psychosocial burden. Most importantly, by using the HIP questionnaire, this study is the first to identify the origins of psychosocial burden of GW patients for both genders. We found that female patients with GW had heavier worries and concerns than male patients, but men had heavier psychosocial burden regarding domains of self-image and interaction with doctors. The origins of the psychosocial burden were mostly from "Self Image" and "Sexual Impact" domains, which highlighted the key domains that doctors should focus on. Results from this study will be informative for prevention of GW and control efforts in China and other similar settings.

\section{Predominant domain of psychosocial burden for patients} with GW

It has been evidenced that patients with GW lost quality of life and had significantly heavy psychosocial burden 


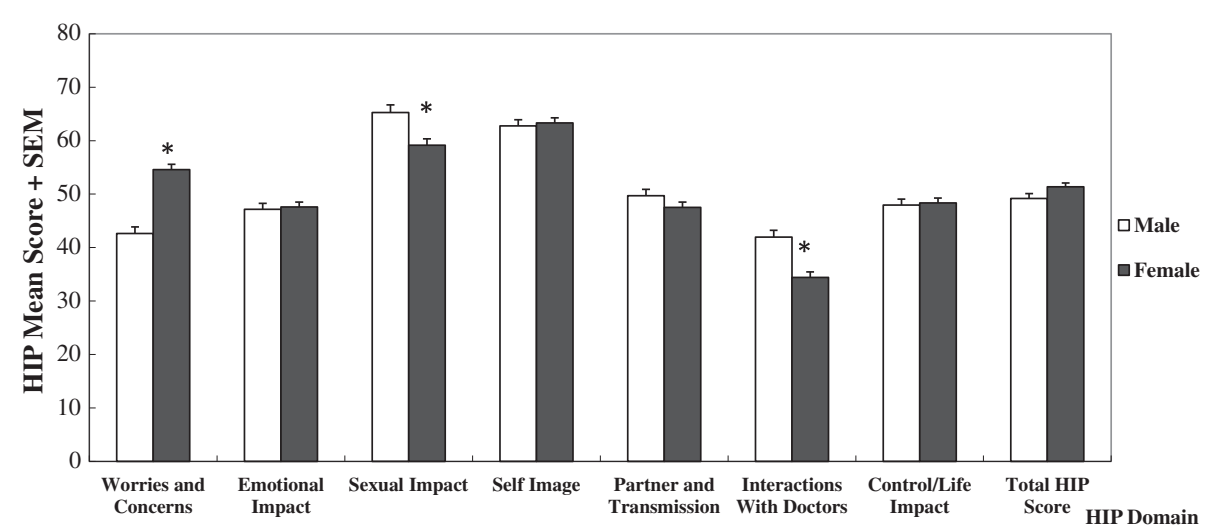

Figure 1 The overall HPV Impact Profile (HIP) score means and Std. Errors, by individual domain and genders. Note: Multivariate analysis: adjusted for age, education, income and insurance coverage; ${ }^{*} P<0.001$.

by various measures such as EQ-5D, group interview, and the Short-Form (SF)-12 [8,12-14]. However, this study was the first to provide quantitative assessment for different psychosocial domains of Chinese GW patients of both genders by HIP questionnaire. Our results would help investigate the sources of the burden and provide accompanying suggestions to relieve it. Based on our data, the sexually related domains impacted patients the most. Specifically, the "Sexual Impact" domain represented the frequency and satisfaction of sexual life, and "Self Image" domain represented feelings of attractiveness or lack thereof, shame, and disgust. These two domains showed the most significant psychosocial burden, which suggested that self-identification and sexual function were the most significant issues that patients considered. The other sexually related domain "Partner and Transmission" also showed moderate psychosocial burdens, which represented partner acceptance and concern of transmission to/from partners. Our results were supported by the UK study, which indicated that women with GW suffered most in the three domains of sexual impact (63.2), self-image (62.7), and partner issues and transmission (58.7) [15]. These three significantly represented domains partly reflect the ingrained conception in a culturally conservative China that STIs, such as genital warts implies promiscuity. The highly-visible and recurrent nature of genital warts may directly and negatively impact libido and sexual function, possibly leading to significant psychosocial burden of negative self-image and consternation for both patient and partner.

\section{Comparison among different countries and areas}

Generally, we found that the psychosocial burden of Chinese patients with GW was heavy, with the HIP mean score of 50.49, 49.20 and 51.38 for GW patients in total, male patients, and female patients, respectively. Compared with other settings that also used HIP questionnaire for the evaluation of psychosocial burden, Chinese patients showed comparable HIP score with that of British patients (50.9), but higher scores than those of Australians (45.7) and lower scores than those of Taiwanese patients (62.5) [6,7,15]. Different social development and cultures may lead to this variety. Even within the mainland of China, we found differences

Table 3 HIP Scores for participants by gender/ HIP domains

\begin{tabular}{|c|c|c|c|c|c|c|}
\hline \multirow[t]{2}{*}{ Domain } & \multicolumn{2}{|c|}{ Total* } & \multicolumn{2}{|c|}{ Male* } & \multicolumn{2}{|c|}{ Female* } \\
\hline & HIP score & $95 \% \mathrm{Cl}$ & HIP Score & $95 \% \mathrm{Cl}$ & HIP Score & $95 \% \mathrm{Cl}$ \\
\hline Worries and concerns & 49.72 & $48.12-51.32$ & 42.62 & $40.16-45.07$ & 54.57 & $52.56-56.58$ \\
\hline Emotional impact & 47.40 & $46.00-48.80$ & 47.14 & $44.88-49.40$ & 47.58 & $45.73-49.43$ \\
\hline Sexual impact & 61.64 & $59.87-63.41$ & 65.26 & $62.43-68.10$ & 59.16 & $56.85-61.48$ \\
\hline Self image & 63.09 & $61.67-64.50$ & 62.77 & $60.48-65.05$ & 63.31 & $61.43-65.18$ \\
\hline Partner and transmission & 48.42 & $46.96-49.87$ & 49.71 & $47.37-52.06$ & 47.53 & $45.61-49.45$ \\
\hline Interactions with doctors & 37.47 & 35.89-39.05 & 41.97 & $39.47-44.47$ & 34.40 & $32.35-36.44$ \\
\hline Control/life impact & 48.19 & $46.87-49.51$ & 47.96 & $45.82-50.09$ & 48.35 & $46.61-50.10$ \\
\hline Total HIP score & 50.49 & $49.41-51.57$ & 49.20 & $47.46-50.94$ & 51.38 & $49.95-52.80$ \\
\hline
\end{tabular}

*Adjusted for age, insurance coverage, level of education and income. 


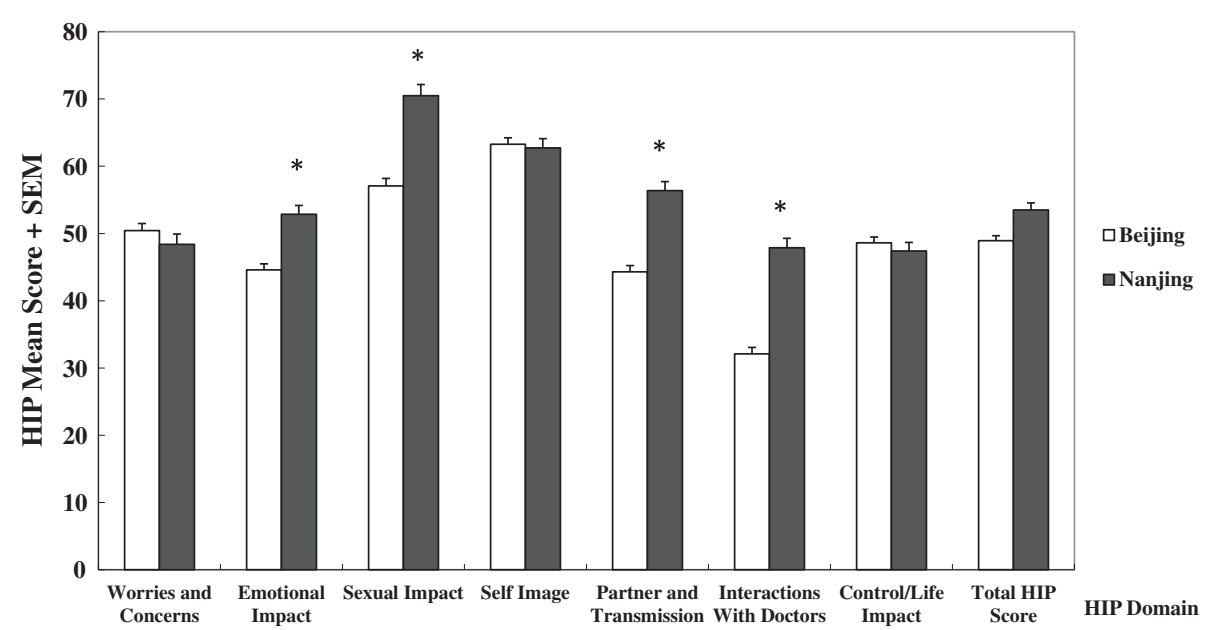

Figure 2 The overall HPV Impact Profile (HIP) score means and Std. Errors, by individual domain and settings (Beijing vs. Nanjing). Note: Multivariate analysis: adjusted for age, education, income and insurance coverage; ${ }^{*} P<0.001$.

between regions. Women in Nanjing (59.8) suffered a higher psychosocial burden both than that of the average national (52.2) and urban levels (53.2) [5]. Specifically, we found that patients in Nanjing suffered heavier psychosocial burdens than those of Beijing within the following four domains: "Emotional Impact", "Sexual Impact", "Partner and Transmission", and "Interactions with Doctors". According to our data, most patients in Nanjing were from rural areas of the North Jiangsu and Anhui Province, and they had lower levels of educational and less knowledge about HPV-related diseases. This may partly contribute to the psychosocial burden.

\section{Different psychosocial impact of GW between genders}

Generally, women experienced a heavier psychosocial burden than men, which was also identified in a previous UK study using EQ-5D questionnaires [4]. This study specified domains resulting to the difference. Women had a significantly heavier psychosocial burden than men within the "Worries and Concern" domain, but a lower psychosocial burden within domains of "Sexual Impact" and "Interactions with Doctors".

Heavier worries and concern among women may stem from the following aspects. Firstly, Traditional Chinese culture requires women to be elegant, virtuous, and obey their husbands, Women are subordinate in most Chinese families, the dependence on their husband leads to be more introverted, sentimental and emotionally sensitive. Secondly, feelings of disgrace, discomfort, and shame from the GW may have women suspect extramarital affairs, and finally lead to emotive crises between couples. Another possible reason is fear of future fertility and risk of cervical cancer. However, men had more psychosocial pressure than women in domains of "Sexual Impact" and "Interactions with Doctors". For the sexual component, a possible reason is that the dominant role in sexual activity leads men to care more about their sexual function and the ability to satisfy their partner. For "Interactions with Doctors" domain, men may be less communicative and unwilling to express their pain to doctors. Moreover, most of the doctors were women in this study, which may also lead to a more negative response from men by gender embarrassment.

\section{Suggestions for alleviation of psychosocial burdens}

We suggest developing the following methods to help healthcare providers alleviate these psychosocial burdens in addition to the current basic biological medical treatments in China.

First, the administration of hospitals and physicians should work together to help change the current biomedical therapy model to a bio-psycho-social model. An investigation in Wuhan showed that only $22.4 \%$ of general hospitals offered mental health services, among which $76.5 \%$ were in the top-level hospitals [16]. A formal system for "Medical-psychological counseling" should be established in comprehensive hospitals, including establishing special psychological counseling departments and providing essential financial support for consultation. In addition, appropriate training for the identification of psychosocial disease should be developed for Chinese medical students and physicians, as this is currently lacking.

Secondly, we know that as a developing country, there is a huge gap between basic medical therapy and the demand of patients in China. To help alleviate the psychosocial burden, we suggest the development of innovative approaches to help with consultation. There are tiered levels of medical workers in China, including the provincial, municipal, county, and village levels. Although these 
medical workers receive different levels of formal medical training, even the lesser-trained workers usually function as physicians within their local communities, gaining trust and respect from their patients. We suggest physicians and other medical workers work together to provide educational lectures and counseling services during the primary stages of the aforementioned consultation system establishment. In addition, we suggest that doctors not only focus their discussion with patients on the recurrent nature and hard-to-cure status when consulted for GW, but also provide patients effective preventable measures and lead them to local medical workers for helpful consultation [5]. The providerpatient relationship is a delicate one, and a compassionate, intelligent approach to treatment and prevention is imperative. STIs are sensitive subjects to discuss due to harsh stigmatization, and it would behoove health care providers to treat these situations as such. As for educational measures, local medical workers might also put up posters in the community and hold discussion sessions to increase awareness of disease etiology and preventive measures.

\section{Study limitations}

Firstly, selection biases could occur in this study due to the hospital-based, convenient sampling method we used; we also failed to assess the differences in characteristics between the study participants and those who declined to participate due to lacking of information of non-participants. Secondly, the cross-sectional data is limited for the time-dependent trend of psychosocial burdens of genital warts. Additional follow-up assessments should focus on multiple longitudinal time points after diagnoses, to help identify domains with sustainable impact and prioritize contents for consultation. Thirdly, the sample size was not large enough to acquire a high level of reliability, yet our data still provides novel information regarding specific HPV-related negative impacts of GW.

\section{Conclusions}

In conclusion, patients with genital warts had heavier psychosocial burdens than those of the general population, with females bearing a heavier burden than males. Self-image and sexual function were issues with which study patients experienced the highest psychosocial burdens. The distinctions of origins of psychosocial burdens between genders also help to prioritize causes of psychosocial burden and provide targeted psychosocial consultation services of GW. This study will also provide baseline utility data for a more detailed costeffectiveness analysis of prophylactic HPV vaccination, which could help guide the development of educational public health campaigns.

\section{Additional file}

\section{Additional file 1: HPV IMPACT PROFILE.}

\section{Abbreviations}

GW: Genital warts; HPV: Human papillomavirus; HIP: HPV Impact Profile; QoL: Quality of life; STD: Sexual transmitted diseases; STI: Sexually transmitted Infections.

\section{Competing interests}

All author's declare that they have no competing interests.

\section{Authors' contributions}

SZ Qi and SM Wang analyzed the data, generated the tables, figures and wrote the manuscript. JF Shi made important contributions on the result explanation sections and field work. QQ Wang and XS Chen made substantial contributions to the result interpretation. $L J$ Sun, A Liu and XL Xu made important contributions to the field work and data collection. Ning Jiang contributed to the data analysis and helped interpret the data in a meaningful way. N Zhang and P Sivasubramaniam helped review and revise the manuscript. YL Qiao helped design the study and made comments regarding the intellectual content. All authors read and approved the final manuscript.

\section{Acknowledgments}

This study was funded by Merck Sharp \& Dohme Ltd (China). We thank the all the doctors and the patients who participated in our study, as well as the colleagues of Cancer Institute/Hospital, Chinese Academy of Medical Sciences, Peking Union Medical College and STD Clinic department of Chinese Academy of Medical Sciences of Peking Union Medical College for their generous support.

\section{Author details}

${ }^{1}$ Institute of Dermatology, Chinese Academy of Medical Sciences, Peking Union Medical College, Nanjing, China. ${ }^{2}$ Cancer Institute/Hospital, Chinese Academy of Medical Sciences, Peking Union Medical College, Beijing, China. ${ }^{3}$ National Center for STD Control, China CDC, Nanjing, China. ${ }^{4}$ Beijing You An Hospital, Capital Medical University, Beijing, China. ${ }^{5}$ University of California, Los Angeles, USA. ${ }^{6}$ Vanderbilt University School of Medicine, Nashville, Tennessee, USA.

Received: 14 March 2014 Accepted: 26 June 2014 Published: 21 July 2014

\section{References}

1. Koshiol JE, Laurent SA, Pimenta JM: Rate and predictors of new genital warts claims and genital warts-related healthcare utilization among privately insured patients in the United States. Sex Transm Dis 2004, 31(12):748-752.

2. Patel RV, Yanofsky VR, Goldenberg G: Genital warts: a comprehensive review. J Clin Aesthet Dermatol 2012, 5(6):25-36.

3. Garland SM, Hernandez-Avila M, Wheeler CM, Perez G, Harper DM, Leodolter S, Tang GW, Ferris DG, Steben M, Bryan J, Taddeo FJ, Railkar R, Esser MT, Sings HL, Nelson M, Boslego J, Sattler C, Barr E, Koutsky LA, Females United to Unilaterally Reduce Endo/Ectocervical Disease (FUTURE) I Investigators: Quadrivalent vaccine against human papillomavirus to prevent anogenital diseases. N Engl J Med 2007, 356(19):1928-1943.

4. Woodhall S, Ramsey T, Cai C, Crouch S, Jit M, Birks Y, Edmunds WJ, Newton $\mathrm{R}$, Lacey $\mathrm{CJ}$ : Estimation of the impact of genital warts on health-related quality of life. Sex Transm Infect 2008, 84(3):161-166.

5. Wang SM, Shi JF, Kang DJ, Song P, Qiao YL: Impact of human papillomavirusrelated lesions on quality of life: a multicenter hospital-based study of women in Mainland China. Int J Gynecol Cancer 2011, 21(1):182-188.

6. Pirotta M, Ung L, Stein A, Conway EL, Mast TC, Fairley CK, Garland S: The psychosocial burden of human papillomavirus related disease and screening interventions. Sex Transm Infect 2009, 85(7):508-513.

7. Wang $K L$, Jeng CJ, Yang YC, Chen CA, Cheng WF, Chen TC, Mast TC, Wang YC, Hsieh CY: The psychological impact of illness among women experiencing human papillomavirus-related illness or screening interventions. J Psychosom Obstet Gynaecol 2010, 31(1):16-23. 
8. Shi JF, Kang DJ, Qi SZ, Wu HY, Liu YC, Sun LJ, Li L, Yang Y, Li Q, Feng XX Zhang LQ, Li J, Li XL, Yang Y, Niyazi M, Xu AD, Liu JH, Xiao Q, Li LK, Wang $X Z$, Qiao YL: Impact of genital warts on health related quality of life in men and women in mainland China: a multicenter hospital-based cross-sectional study. BMC Public Health 2012, 12:153.

9. Wen Chen KZ, Jufang S, Jane L, Lihong C, Xun Z, Bin L, Ivy W, Yuping W, Youlin Q: Chinese HPV typing group: HPV type-distribution on genital warts in china: a multi-center study. Malmo, Sweden: 25th International Papillomavirus Conference; 2009:30-41.

10. Mast TC, Zhu X, Demuro-Mercon C, Cummings HW, Sings HL, Ferris DG: Development and psychometric properties of the HPV Impact Profile (HIP) to assess the psychosocial burden of HPV. Curr Med Res Opin 2009, 25(11):2609-2619.

11. Bullinger M, Alonso J, Apolone G, Leplege A, Sullivan M, Wood-Dauphinee S, Gandek B, Wagner A, Aaronson N, Bech P, Fukuhara S, Kaasa S, Ware JE Jr: Translating health status questionnaires and evaluating their quality: the IQOLA Project approach. International Quality of Life Assessment. J Clin Epidemiol 1998, 51(11):913-923.

12. Woodhall SC, Jit M, Soldan K, Kinghorn G, Gilson R, Nathan M, Ross JD, Lacey CJ: The impact of genital warts: loss of quality of life and cost of treatment in eight sexual health clinics in the UK. Sex Transm Infect 2011, 87(6):458-463.

13. Senecal M, Brisson M, Maunsell E, Ferenczy A, Franco EL, Ratnam S, Coutlee F, Palefsky JM, Mansi JA: Loss of quality of life associated with genital warts: baseline analyses from a prospective study. Sex Transm Infect 2011, 87(3):209-215.

14. Drolet M, Brisson M, Maunsell E, Franco EL, Coutlee F, Ferenczy A, Ratnam S, Fisher W, Mansi JA: The impact of anogenital warts on health-related quality of life: a 6-month prospective study. Sex Transm Dis 2011, 38(10):949-956.

15. Dominiak-Felden G, Cohet C, Atrux-Tallau S, Gilet H, Tristram A, Fiander A: Impact of human papillomavirus-related genital diseases on quality of life and psychosocial wellbeing: results of an observational, healthrelated quality of life study in the UK. BMC Public Health 2013, 13(1):1065.

16. Ge Q, Wu JL: Study on situation of mental health personnel in the clinics of general hospitals of Wuhan. Master Degree thesis of HUST 2008, 1(1):5-7.

doi:10.1186/1471-2458-14-739

Cite this article as: Qi et al:: Human papillomavirus-related psychosocial impact of patients with genital warts in China: a hospital-based cross-sectional study. BMC Public Health 2014 14:739.

\section{Submit your next manuscript to BioMed Central and take full advantage of:}

- Convenient online submission

- Thorough peer review

- No space constraints or color figure charges

- Immediate publication on acceptance

- Inclusion in PubMed, CAS, Scopus and Google Scholar

- Research which is freely available for redistribution 